\title{
Potencial alelopático de macrófitas aquáticas de um estuário cego ${ }^{1}$
}

\author{
Leandro Kenji Takao ${ }^{2,3}$, Jose Pedro Nepomuceno Ribeiro² e Maria Inês Salgueiro Lima
}

Recebido em 7/04/2010. Aceito em 23/03/2011

\begin{abstract}
RESUMO
(Potencial alelopático de macrófitas aquáticas de um estuário cego). Macrófitas aquáticas representam uma das comunidades mais produtivas e através de sua atividade metabólica são capazes de produzir grandes interferências no ambiente. As interações alelopáticas são aparentemente aumentadas sob condições de estresse biótico e abiótico e podem existir em estuários devido à competição, variações de salinidade e outros fatores. O objetivo desse trabalho foi avaliar as propriedades alelopáticas de extratos aquosos foliares de 25 espécies de macrófitas aquáticas de um estuário cego. Testamos os efeitos dos extratos foliares em quatro concentrações sobre a germinação de alface. Ordenamos e comparamos as espécies doadoras de acordo com a dose reposta sobre a variedade de tratamentos a partir de valores únicos de índices alelopáticos. Onze das 25 espécies diminuíram a porcentagem de germinação, todas diminuíram a velocidade de germinação e aumentaram a entropia informacional de germinação das sementes da espécie alvo em pelo menos uma das concentrações testadas. Crinum americanum L., Sagittaria montevidensis Cham. \& Schl. e Ipomoea cairica (L) Sweet apresentaram os maiores valores de índice alelopático. Em geral, as menores porcentagens de germinação coincidiram com as menores velocidades e maiores entropias informacionais de germinação das sementes de alface, mostrando um conjunto de alterações ocorrendo simultaneamente com o aumento da concentração dos extratos.
\end{abstract}

Palavras-chave: alelopatia, germinação, índice alelopático, Lactuca sativa, ranqueamento

\begin{abstract}
(Allelopathic potential of aquatic macrophytes from a blind estuary). Aquatic macrophytes represent one of the most productive communities and through metabolic activity are capable of producing great interference in the environment. Allelopathic interactions are apparently increased under biotic and abiotic stress and may exist in estuaries due to competition, salinity variation and other factors. The aim of this study was to evaluate the allelopathic properties of leaf extracts of 25 aquatic macrophyte species from a blind estuary. We tested leaf extract effects in four concentrations on the germination of lettuce. We ranked and compared the donor species according to the dose-response over the variety of treatments through unique values of allelopathic indexes. Eleven of 25 species decreased the germination percentage, all of them decreased the speed of germination and increased the germination informational entropy of the target species seeds in at least one of the tested concentrations. Crinum americanum L., Sagittaria montevidensis Cham. \& Schl. and Ipomoea cairica (L.) Sweet presented the highest allelopathic index values. In general, the lowest germination percentages concurred with the lowest germination speed and highest germination informational entropies of lettuce seeds, showing an assemblage of alterations occurring simultaneously with the increase of extract concentrations.
\end{abstract}

Key words: allelopathic index, allelopathy, germination, Lactuca sativa, ranking

\section{Introdução}

Alelopatia é definida como a capacidade de uma planta influenciar o desenvolvimento de outra planta ou microorganismo (Rice 1984). Qualquer órgão vegetal é capaz de produzir substâncias alelopáticas (Putnam \& Tang 1986), e a alelopatia é um fator importante na determinação das interações bióticas (Blanco 2007). Ela influencia a sucessão vegetal, a dominância, a formação clímax, a diversidade de espécies, a estrutura das comunidades e a produtividade, sendo importante em ambientes naturais e agroecossistemas (An 2005). Processos alelopáticos também ocorrem em ambientes aquáticos, e todos os grupos de produtores primários possuem espécies capazes de produzir e liberar compostos alelopaticamente ativos (Gross 2003). Em ambientes aquáticos, o processo alelopático é diferenciado. Na água, os aleloquímicos movimentam-se com uma velocidade maior do que no solo (Ferreira \&

\footnotetext{
1 Parte da dissertação de Mestrado do primeiro Autor

2 Universidade Federal de São Carlos, Programa de Pós-Graduação em Ecologia e Recursos Naturais, Laboratório de Sistemática e Ecologia Química, São Carlos, SP, Brasil

3 Autor para correspondência: lktakao@gmail.com
} 
Aquila 2000). Além disso, a maioria dos fotoautótrofos é cercada por água, e os aleloquímicos liberados pelo organismo doador devem ser suficientemente hidrofílicos e alcançar os organismos receptores em concentrações efetivas apesar da diluição (Gross 2003).

Macrófitas aquáticas representam uma das comunidades mais produtivas dentre todos os ecossistemas, e através do seu metabolismo são capazes de produzir grandes interferências no ambiente (Esteves 1988). Sua atividade alelopática parece ser uma resposta evolutiva eficiente contra fitoplâncton e epífitas (Gross 2003, Erhard \& Gross 2006), uma vez que o sombreamento (frequentemente causado por esses) é o principal fator limitante para as macrófitas (Phillips et al. 1978). Estuário é o ambiente de mistura entre as águas doce e salgada oriundas de um rio e de um oceano (Wolanski 2007), sendo um ambiente intimamente dependente da maré. Dessa maneira, sua flora está constantemente exposta a ciclos de alagamento e salinidade, que estão entre os principais estresses para a vida vegetal. Existem evidências de que condições de estresse biótico ou abiótico aumentam as interações alelopáticas (Gross 2003). Estuários cegos apresentam conexão intermitente entre o rio e o oceano, sendo ambientes bastante imprevisíveis. A imprevisibilidade das condições ambientais é também um estresse severo para plantas aquáticas (Otte 2001), o que sugere que estuários cegos sejam particularmente estressantes para flora, e conseqüentemente, que possuam um número alto de espécies alelopáticas.

Experimentos em laboratório são amplamente utilizados para detectar a atividade biológica de produtos naturais (e.g. atividade aleloquímica) (Hoagland \& Williams 2003). Porém, os bioensaios de germinação de sementes possuem pouca padronização. A maioria das pesquisas foca sobre poucas espécies, principalmente espécies agricultáveis para analisar aspectos bioquímicos e fisiológicos da germinação (Aliotta et al. 2006). Os trabalhos sobre alelopatia, por exemplo, empregam metodologias e espécies receptoras variáveis, o que torna difícil a comparação entre as espécies doadoras. Há trabalhos sobre alelopatia para sete das 25 espécies escolhidas para este estudo: Brachiaria mutica (Forsk) Stapf (Chou 1989), Crinum americanum L. (Ribeiro et al. 2009), Eleocharis interstincta (Vahl) Roem. \& Schult. (Sutton \& Portier 1991), Eleocharis flavescens (Poir.) Urb., Eleocharis montana (Kunth) Roem. \& Schult. (Wooten \& Elakovich 1991), Ipomoea cairica (L.) Sweet (Ma et al. 2009), e Typha dominguensis Pers. (Gallardo et al. 1998). Todos esses trabalhos demonstram efeitos inibitórios. Entretanto, como eles empregaram metodologias e espécies alvo distintas, a comparação direta entre eles não é possível. A comparação dos potenciais fitotóxicos das espécies doadoras em bioensaios de germinação se torna uma ferramenta para a seleção de espécies promissoras aos estudos nesta área. A alface é uma espécie sensível a diversas categorias e concentrações de metabólitos secundários, sendo assim uma espécies alvo amplamente utilizada como bioindicadora nesse tipo de experimento (Ferreira \& Aquila 2000). Essas características tornam a alface particularmente útil para o ranqueamento do potencial alelopático das espécies.

Dessa forma, o objetivo desse trabalho foi avaliar as propriedades alelopáticas de extratos foliares aquosos de 25 espécies de macrófitas aquáticas do estuário cego do Rio Massaguaçu sobre a germinação de alface. Sendo este um ambiente abioticamente estressante, tanto do ponto de vista dos ciclos de alagamento quanto da presença de sal, esperamos que exista um número grande espécies com potencial alelopático.

\section{Material e métodos}

O local de coleta, o estuário do Rio Massaguaçu (Ca-

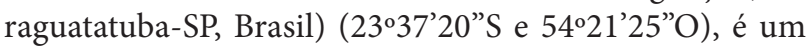
estuário cego, separado do oceano por uma barra de areia. Em março de 2009, coletamos de forma assistemática folhas adultas, sem sinais de predação ou doenças, de 25 espécies de macrófitas aquáticas em diferentes estágios fenológicos (Tab. 1). Secamos as folhas à $45^{\circ} \mathrm{C}$ em estufa de circulação forçada até a estabilização das massas. Trituramos em moinho e estocamos a $-10^{\circ} \mathrm{C}$ em bolsas plásticas até o uso. Preparamos extratos aquosos $10 \%(\mathrm{p} / \mathrm{v})$ com os pulverizados das folhas secas e água destilada. Colocamos por 5 minutos em agitador magnético $\left(22^{\circ} \mathrm{C}\right)$ e após acondicionamento à $6^{\circ} \mathrm{C}$ durante 12 horas, filtramos a vácuo em papel filtro (3 $\mu \mathrm{m})$. Fizemos diluições de 5, 2,5 e 1,25\% em água destilada a partir da solução resultante (10\%) e testamos os seus efeitos sobre a germinação de alface (Lactuca sativa L., cultivar Grand Rapids). Os vouchers das espécies testadas foram depositados no Herbário do Departamento de Botânica da Universidade Federal de São Carlos.

\section{Bioensaio de germinação}

Colocamos trinta sementes de alface (cipselas que neste trabalho serão usadas como sinônimo de sementes; adquiridas em loja especializada) em placa de Petri $(9 \mathrm{~cm}$ de diâmetro) contendo camada dupla de papel filtro (3 $\mu \mathrm{m}$ ) umedecida com $5 \mathrm{~mL}$ de solução. Utilizamos água destilada como controle. Lacramos todas as placas de Petri com filme PVC, fechamos e colocamos em estufa DBO $\left(25^{\circ} \mathrm{C}\right.$ e $12 \mathrm{~h}-12 \mathrm{~h}$ de luz-escuro). Fizemos leituras a cada $12 \mathrm{~h}$ considerando germinadas sementes com protrusão radicular igual ou superior a $2 \mathrm{~mm}$ (Brasil 1992). Após 10 dias calculamos a porcentagem, velocidade e entropia informacional (E) de germinação (Labouriau 1983). Calculamos a área de inibição entre a resposta do controle e a curva de dose-resposta (resposta da espécie alvo) gerada pela concentração das soluções (An 2005). Obtivemos um único valor de potencial alelopático para cada espécie, o índice alelopático (IA). A partir desses valores, comparamos e ranqueamos as espécies segundo seu potencial alelopático sobre cada um dos parâmetros analisados. Para o cálculo 
Tabela 1. Porcentagens de germinação de Lactuca sativa L. sob ação de extratos em diferentes concentrações das espécies doadoras e respectivos Índices Alelopáticos (IAs). Letras iguais não diferem entre si pela ANOVA e pós-teste de Tukey. Asterisco: diferença significativa em relação ao controle pelo teste de Mann-Whitney $(\mathrm{p}<0,05)$ dentro da mesma linha. n: infértil. s: fértil (florido ou com soros). 0,0: controle. As espécies foram dispostas do maior para o menor IA.

\begin{tabular}{|c|c|c|c|c|c|c|c|c|c|}
\hline \multirow{2}{*}{ Espécie } & \multirow{2}{*}{ Família } & \multirow{2}{*}{ Fértil } & \multicolumn{5}{|c|}{ Concentração (\%) } & \multirow{2}{*}{\multicolumn{2}{|c|}{ IA }} \\
\hline & & & 0 & 1,25 & 2,5 & 5 & 10 & & \\
\hline Crinum americanum $\mathrm{L}$. & Amaryllidaceae & $n$ & 97,3 & 95,3 & 94,0 & $48,0^{*}$ & $0,0^{*}$ & 435,8 & A \\
\hline Sagittaria montevidensis Cham. \& Schl. & Alismataceae & $n$ & 97,3 & 92,7 & 94,7 & $81,3^{*}$ & $0,6^{*}$ & 309,5 & $\mathrm{AB}$ \\
\hline Ipomoea cairica (L) Sweet & Convolvulaceae & $s$ & 97,3 & 94,7 & 91,3 & $86,6^{*}$ & $28,3^{*}$ & 208,8 & $\mathrm{BC}$ \\
\hline Eleocharis flavescens (Poir.) Urb. & Cyperaceae & $s$ & 97,3 & 94,0 & 96,7 & 96,0 & $66,6^{*}$ & 117,5 & $\mathrm{CD}$ \\
\hline Ludwigia erecta (L.) H. Hara & Onagraceae & $n$ & 97,3 & 96,0 & 94,0 & 95,3 & $62,0^{*}$ & 102,9 & $\mathrm{CDE}$ \\
\hline Acrostichum danaeifolium Langsd \& Fisch & Pteridaceae & $s$ & 97,3 & 95,3 & 94,7 & 94,7 & $73,3^{*}$ & 101,2 & $\mathrm{CDE}$ \\
\hline Brachiaria mutica (Forsk) Stapf & Poaceae & $s$ & 97,3 & 95,0 & 93,3 & 92,7 & $73,3^{*}$ & 91,1 & $\mathrm{CDEF}$ \\
\hline Bacopa monnieri (L.) Wettst. & Scrophulariaceae & $s$ & 97,3 & 98,0 & 92,7 & 96,0 & $55,3^{*}$ & 82,2 & $\mathrm{CDEF}$ \\
\hline Ludwigia hyssopifolia (G. Don) Exel & Onagraceae & $s$ & 97,3 & 98,0 & 98,7 & 96,0 & $65,3^{*}$ & 82,0 & CDEF \\
\hline Typha dominguensis Pers. & Typhaceae & $n$ & 97,3 & 96,7 & 97,3 & 93,3 & $86,6^{*}$ & 42,0 & DEF \\
\hline Echinochloa polystachya (Kunth) Hitchc & Poaceae & $s$ & 97,3 & 93,3 & 92,7 & 93,3 & 91,3 & 41,2 & DEF \\
\hline Thelypteris interrupta (Willd.) Iwats. & Thelypteridaceae & $n$ & 97,3 & 98,0 & 96,0 & 96,0 & $88,0^{*}$ & 30,4 & DEF \\
\hline Hymenachne amplexicaulis (Rudge) Ness. & Poaceae & $s$ & 97,3 & 98,0 & 98,0 & 94,0 & 90,7 & 30,0 & DEF \\
\hline Ludwigia octovalvis (Jacq.) Raven & Onagraceae & $s$ & 97,3 & 94,7 & 93,3 & 95,3 & 90,0 & 27,1 & DEF \\
\hline Schoenoplectus californicus (C.A. Mey.) Soják & Cyperaceae & $s$ & 97,3 & 96,7 & 97,3 & 96,0 & 88,7 & 27,0 & DEF \\
\hline Scleria latifolia Sw. & Cyperaceae & $n$ & 95,3 & 98,0 & 93,3 & 94,0 & 92,7 & 27,0 & DEF \\
\hline Acroceras zizanioides (HBK) Dandy & Poaceae & $s$ & 97,3 & 96,7 & 98,7 & 93,3 & 94,0 & 21,2 & DEF \\
\hline Eleocharis interstincta (Vahl) Roem. \& Schult. & Cyperaceae & $s$ & 97,3 & 98,7 & 96,7 & 95,3 & 94,0 & 14,5 & DEF \\
\hline \multirow[t]{2}{*}{ Eleocharis minima Kunth } & Cyperaceae & $n$ & 97,3 & 96,7 & 96,7 & 96,0 & 94,7 & 13,3 & DEF \\
\hline & & & & & & & & 0,0 & DEF \\
\hline Scleria mitis O. Berg & Cyperaceae & $s$ & 95,3 & 98,7 & 94,0 & 96,0 & 96,7 & $-5,4$ & DEF \\
\hline Eleocharis montana (Kunth) Roem. \& Schult. & Cyperaceae & $s$ & 97,3 & 98,7 & 95,3 & 95,3 & 99,3 & $-9,9$ & DEF \\
\hline Polygonum ferrugineum Wedd. & Polygonaceae & $s$ & 95,3 & 96,0 & 98,7 & 96,0 & 96,7 & $-12,5$ & $\mathrm{EF}$ \\
\hline Calyptrocarya longifolia (Rudge) Kunth & Cyperaceae & $s$ & 95,3 & 92,7 & 97,3 & 94,7 & 97,3 & $-18,3$ & $\mathrm{EF}$ \\
\hline Polygonum hydropiperoides Michx. & Polygonaceae & $s$ & 95,3 & 99,3 & 96,7 & 97,3 & 95,3 & $-20,8$ & $\mathrm{EF}$ \\
\hline Polygonum meisnerianum Cham. \& Schltdl. & Polygonaceae & $s$ & 95,3 & 97,3 & 96,7 & 96,7 & 98,0 & $-30,4$ & $\mathrm{~F}$ \\
\hline
\end{tabular}

do IA da entropia informacional utilizamos os valores de $\mathrm{E}^{-1}$ para que tratamentos com germinabilidade nula não fossem subestimados.

Medimos o $\mathrm{pH}$ e os potenciais osmóticos dos extratos e montamos bioensaios de germinação, submetendo as sementes de alface a soluções de Polietilenoglicol 6000, soluto mais utilizado para verificar a influência desse fator por ser quimicamente inerte e não apresentar toxicidade sobre as sementes (Villela et al. 1991), com os valores de potenciais osmóticos - 0,$1 ;-0,2 ;-0,3 ;-0,4 ;-0,5 ;-0,6 ;-0,7 ;-0,8 ;-0,9,-1,0$.

Aplicamos cinco tratamentos para cada espécie doadora (controle e soluções $1,25,2,5,5,10 \%$ ) em delineamento experimental de blocos casualizados com cinco réplicas. Fizemos a análise da normalidade dos dados pelo teste de Kolmogorov-Smirnov. Baseado no resultado do teste de normalidade, analisamos os dados de porcentagem de germinação através do teste de Mann-Whitney $(\mathrm{p}<0,05)$ e os dados de velocidade, entropia informacional de germinação e índices alelopáticos pela ANOVA com pós-teste de Tukey, através do programa estatístico GraphPad Prism.

\section{Resultados e discussão}

Observamos alterações significativas sobre todas as variáveis da germinação (porcentagem, velocidade e entropia informacional) das sementes de alface submetidas à ação dos extratos foliares. Extratos de onze espécies de 
macrófitas apresentaram efeito inibitório significativo sobre a porcentagem de germinação em pelo menos uma concentração, sendo que Crinum americanum L., Sagittaria montevidensis Cham. \& Schl. e Ipomoea cairica (L.) Sweet, apresentaram os maiores valores de IA (Tab. 1). Estas diminuíram significativamente a porcentagem de germinação nas concentrações 5 e 10\%. Eleocharis flavescens (Poir.) Urb., Ludwigia erecta (L.) H. Hara, Acrostichum danaeifolium Langsd \& Fisch, Brachiaria mutica (Forsk) Stapf, Bacopa monnieri (L.) Wettst., Ludwigia hyssopifolia (G. Don) Exel, Typha dominguensis Pers. e Thelypteris interrupta (Willd.) Iwats. diminuíram a porcentagem de germinação apenas na concentração máxima dos extratos. Muitas vezes a atividade alelopática não ocorre sobre a germinabilidade, mas sobre outras variáveis do processo germinativo (Ferreira \&
Aquila 2000). A alface, apesar de ser uma espécie sensível, apresenta um processo de germinação muito rápido. Em 24 horas quase todas as sementes já haviam germinado. Assim, o efeito na germinabilidade final pode ser muito sutil. De fato, a porcentagem de germinação das sementes de alface foi a variável menos sensível em nossos bioensaios. A maioria das espécies testadas (14 de 25) não apresentou efeito estatisticamente significativo sobre este parâmetro (Tab. 1).

A velocidade de germinação foi o parâmetro mais sensível à ação dos extratos aquosos. Todas as espécies afetaram negativamente a velocidade em pelo menos uma das concentrações testadas (Tab. 2). Observamos IAs significativos sobre a velocidade de germinação em relação ao controle em dez espécies, sendo que $C$. americanum e $S$. montevidensis reduziram significativamente a velocidade de

Tabela 2. Velocidades de germinação de Lactuca sativa L. sob ação de extratos em diferentes concentrações das espécies doadoras e respectivos Índices Alelopáticos (IAs). Letras minúsculas iguais não diferem entre si dentro da mesma linha e letras maiúsculas iguais não diferem entre si, pela ANOVA e pós-teste de Tukey $(\mathrm{p}<0,05)$. $\mathbf{0 , 0 0}$ : controle. As espécies foram dispostas do maior para o menor IA.

\begin{tabular}{|c|c|c|c|c|c|c|c|c|c|c|c|c|c|}
\hline \multirow{3}{*}{$\begin{array}{l}\text { Espécie } \\
\text { Crinum americanum } \mathrm{L} \text {. }\end{array}$} & \multirow{3}{*}{$\begin{array}{c}\text { Família } \\
\text { Amaryllidaceae }\end{array}$} & \multicolumn{10}{|c|}{ Concentração (\%) } & \multirow{2}{*}{\multicolumn{2}{|c|}{ IA }} \\
\hline & & \multicolumn{2}{|c|}{0} & \multicolumn{2}{|c|}{1,25} & \multicolumn{2}{|c|}{2,5} & \multicolumn{2}{|l|}{5} & \multicolumn{2}{|l|}{10} & & \\
\hline & & 0,036 & a & 0,031 & $\mathrm{~b}$ & 0,026 & $\mathrm{~b}$ & 0,013 & $c$ & 0,000 & $\mathrm{~d}$ & 0,21 & A \\
\hline Sagittaria montevidensis Cham. \& Schl. & Alismataceae & 0,036 & a & 0,027 & $\mathrm{~b}$ & 0,025 & $\mathrm{~b}$ & 0,010 & $c$ & 0,000 & $\mathrm{~d}$ & 0,21 & A \\
\hline Ipomoea cairica (L) Sweet & Convolvulaceae & 0,036 & a & 0,033 & $\mathrm{ac}$ & 0,028 & $\mathrm{bc}$ & 0,018 & $\mathrm{~d}$ & 0,000 & e & 0,18 & $\mathrm{AB}$ \\
\hline Acrostichum danaeifolium Langsd \& Fisch & Pteridaceae & 0,036 & a & 0,041 & a & 0,030 & $\mathrm{~b}$ & 0,017 & $\mathrm{c}$ & 0,009 & $\mathrm{~d}$ & 0,15 & $\mathrm{ABC}$ \\
\hline Ludwigia hyssopifolia (G. Don) Exel & Onagraceae & 0,036 & $\mathrm{ab}$ & 0,045 & a & 0,029 & $\mathrm{bc}$ & 0,020 & $\mathrm{~cd}$ & 0,008 & $\mathrm{~d}$ & 0,14 & $\mathrm{ABCD}$ \\
\hline Ludwigia octovalvis (Jacq.) Raven & Onagraceae & 0,036 & $\mathrm{ab}$ & 0,038 & a & 0,030 & $\mathrm{~b}$ & 0,023 & c & 0,014 & c & 0,14 & $\mathrm{ABCD}$ \\
\hline Hymenachne amplexicaulis (Rudge) Ness. & Poaceae & 0,036 & a & 0,035 & $\mathrm{ab}$ & 0,029 & $\mathrm{bc}$ & 0,023 & c & 0,012 & $\mathrm{~d}$ & 0,13 & $\mathrm{ABCD}$ \\
\hline Ludwigia erecta (L.) H. Hara & Onagraceae & 0,036 & a & 0,037 & a & 0,036 & a & 0,020 & $\mathrm{~b}$ & 0,010 & $\mathrm{~b}$ & 0,13 & $\mathrm{ABCD}$ \\
\hline Eleocharis flavescens (Poir.) Urb. & Cyperaceae & 0,036 & a & 0,035 & a & 0,032 & $\mathrm{ab}$ & 0,026 & $\mathrm{~b}$ & 0,000 & c & 0,12 & $\mathrm{ABCD}$ \\
\hline Thelypteris interrupta (Willd.) Iwats. & Thelypteridaceae & 0,036 & $\mathrm{a}$ & 0,038 & a & 0,033 & a & 0,023 & $\mathrm{~b}$ & 0,012 & c & 0,12 & $\mathrm{ABCD}$ \\
\hline Acroceras zizanioides (HBK) Dandy & Poaceae & 0,036 & a & 0,036 & a & 0,030 & $\mathrm{a}$ & 0,023 & $\mathrm{~b}$ & 0,014 & c & 0,12 & $\mathrm{ABCDE}$ \\
\hline Eleocharis interstincta (Vahl) Roem. \& Schult. & Cyperaceae & 0,036 & a & 0,031 & $\mathrm{ab}$ & 0,029 & $\mathrm{ab}$ & 0,026 & $\mathrm{~b}$ & 0,014 & c & 0,11 & $\mathrm{ABCDE}$ \\
\hline Bacopa monnieri (L.) Wettst. & Scrophulariaceae & 0,036 & a & 0,037 & a & 0,032 & $\mathrm{a}$ & 0,032 & $\mathrm{a}$ & 0,012 & $\mathrm{~b}$ & 0,11 & $\mathrm{ABCDE}$ \\
\hline Scleria latifolia Sw. & Cyperaceae & 0,040 & a & 0,036 & $\mathrm{ab}$ & 0,035 & $\mathrm{ab}$ & 0,031 & $\mathrm{bc}$ & 0,024 & c & 0,09 & $\mathrm{BCDE}$ \\
\hline Polygonum meisnerianum Cham. \& Schltdl. & Polygonaceae & 0,040 & a & 0,039 & a & 0,039 & $\mathrm{a}$ & 0,030 & $\mathrm{~b}$ & 0,020 & c & 0,09 & $\mathrm{BCDE}$ \\
\hline Polygonum hydropiperoides Michx. & Polygonaceae & 0,040 & a & 0,035 & a & 0,036 & $\mathrm{a}$ & 0,033 & $\mathrm{a}$ & 0,017 & $\mathrm{~b}$ & 0,09 & $\mathrm{BCDE}$ \\
\hline Brachiaria mutica (Forsk) Stapf & Poaceae & 0,036 & $\mathrm{abcd}$ & 0,039 & $\mathrm{~b}$ & 0,039 & c & 0,030 & $\mathrm{~d}$ & 0,016 & e & 0,09 & $\mathrm{BCDE}$ \\
\hline Calyptrocarya longifólia (Rudge) Kunth & Cyperaceae & 0,040 & a & 0,037 & $\mathrm{ab}$ & 0,035 & $\mathrm{ab}$ & 0,030 & $\mathrm{bc}$ & 0,027 & c & 0,08 & $\mathrm{BCDE}$ \\
\hline Polygonum ferrugineum Wedd. & Polygonaceae & 0,040 & a & 0,034 & $\mathrm{ab}$ & 0,037 & a & 0,030 & $\mathrm{bc}$ & 0,027 & c & 0,08 & $\mathrm{BCDE}$ \\
\hline Echinochloa polystachya (Kunth) Hitchc & Poaceae & 0,036 & a & 0,040 & $\mathrm{a}$ & 0,035 & $\mathrm{a}$ & 0,028 & $\mathrm{~b}$ & 0,017 & c & 0,08 & $\mathrm{BCDE}$ \\
\hline Typha dominguensis Pers. & Typhaceae & 0,036 & a & 0,038 & a & 0,034 & $\mathrm{ab}$ & 0,030 & $\mathrm{~b}$ & 0,020 & c & 0,07 & BCDE \\
\hline Eleocharis minima Kunth & Cyperaceae & 0,036 & a & 0,037 & a & 0,033 & $\mathrm{ab}$ & 0,029 & $\mathrm{bc}$ & 0,024 & c & 0,06 & $\mathrm{BCDE}$ \\
\hline Schoenoplectus californicus (C.A. Mey.) Soják & Cyperaceae & 0,036 & a & 0,041 & a & 0,039 & $\mathrm{a}$ & 0,035 & $\mathrm{a}$ & 0,011 & $\mathrm{~b}$ & 0,06 & $\mathrm{BCDE}$ \\
\hline Scleria mitis O. Berg & Cyperaceae & 0,040 & a & 0,040 & a & 0,038 & $\mathrm{a}$ & 0,036 & $\mathrm{a}$ & 0,025 & $\mathrm{~b}$ & 0,06 & $\mathrm{CDE}$ \\
\hline \multirow[t]{2}{*}{ Eleocharis montana (Kunth) Roem. \& Schult. } & Cyperaceae & 0,036 & a & 0,037 & a & 0,036 & $\mathrm{a}$ & 0,035 & $\mathrm{a}$ & 0,026 & $\mathrm{~b}$ & 0,03 & $\mathrm{D}$ \\
\hline & & & & & & & & & & & & 0,00 & E \\
\hline
\end{tabular}


germinação em todas as concentrações testadas. O aumento da atividade inibitória com o aumento da concentração do extrato aquoso testado também ocorreu em relação a esse parâmetro em todas as espécies (Tab. 2).

$\mathrm{O}$ aumento da entropia informacional revela a perda da sincronia nas reações metabólicas da germinação indicando heterogeneidade fisiológica nas sementes (Maraschin-Silva \& Aquila 2006). Isso leva a uma distribuição mais esparsa no tempo de germinação dessas sementes. Atribui-se a esse tipo de distribuição um caráter adaptativo, como uma compensação às condições desfavoráveis do meio (Jeller \& Perez 2001). A germinação das sementes de alface foi sincronizada sob condições utilizadas como controle, tendo em geral um pico único de germinação em $24 \mathrm{~h}$. Todas as espécies de macrófitas, exceto Scleria latifolia Sw., afetaram a sincronia de germinação de alface em alguma concentração (Tab. 3). A entropia informacional no processo de germinação da alface apresentou maiores valores sob ação dos extratos de $I$. cairica, S. montevidensis e C. americanum, respectivamente. Observamos maiores valores de entropia na concentração máxima dos extratos em 21 das 25 espécies estudadas. Em A. danaeifolium, S. latifolia, Calyptrocarya longifolia (Rudge) Kunth e Eleocharis minima Kunth não houve este aumento. Nestas, observamos maiores inibições sobre a sincronia de germinação em concentrações intermediárias.

Um conjunto de alterações negativas ocorreu simultaneamente com o aumento da concentração dos extratos. Em geral, as menores porcentagens coincidiram com as menores velocidades e as maiores entropias informacionais da germinação de alface. Alterações nos padrões de germinação podem resultar de efeitos sobre a permeabilidade de membranas, a transcrição e tradução do DNA, o funcionamento dos mensageiros secundários, a respiração por seqüestro de oxigênio (fenóis), a conformação de enzimas e receptores, ou ainda pela combinação destes fatores (Ferreira \& Aquila 2000). Os extratos aquosos são misturas que podem conter substâncias de várias classes como terpenóides, fenólicos, alcalóides, aminoácidos não protéicos, dentre outras. Apresentam assim, efeitos complexos sobre a alface, ainda não elucidados completamente (Maraschin-Silva \& Aquila 2006). O efeito fitotóxico do extrato pode ocorrer pela ação de uma substância isolada ou pela ação sinérgica de várias subtâncias presentes (Macías et al. 1998). Ainda, as substâncias alelopáticas podem atuar em diferentes processos fisiológicos como crescimento e fotossíntese e até mesmo de forma indireta, por intermédio de outros organismos (Ferreira \& Aquila 2000).

A alelopatia pode fornecer uma vantagem competitiva na interação com outros produtores primários nos ambiente aquáticos (Gross 2003). C. americanum foi a espécie que apresentou o maior potencial alelopático, o que nos faz acreditar que essa característica possa ajudar a explicar a abundância e grandes adensamentos dessa espécie verificados no estuário do Rio Massaguaçu. Também identificamos altos potenciais alelopáticos nos extratos de I. cairica e S. montevidensis. No entanto, essas espécies possuem baixas distribuições, sem adensamentos, neste estuário. Os organismos de um mesmo ambiente podem estar adaptados aos aleloquímicos presentes nesse sistema e as interações alelopáticas podem ser mais comuns entre organismos de diferentes ambientes (Reigosa et al. 1999). Dessa forma, os potenciais alelopáticos de $I$. cairica e $S$. montevidensis poderiam favorecê-las, por exemplo, em ambientes nos quais elas são invasoras. De fato, a alelopatia é sugerida como um mecanismo para o sucesso de plantas invasoras (Hierro \& Callaway 2003), e I. cairica é apontada como uma espécie amplamente distribuída pelas regiões tropicais, considerada nociva e invasora extrema (Llamas 2003), e S. montevidensis caracteriza-se como uma planta invasora medianamente freqüente, ocorrendo em ambientes alagados e pantanosos (Lorenzi 2000).

Neste trabalho, os extratos de todas as espécies doadoras afetaram o processo germinativo das sementes de alface. Em geral, a interferência alelopática pode variar dependendo do estágio fenológico do indivíduo doador (Souza Filho et al. 2002) e das condições ambientais (Blanco 2007). Não foi possível coletar todos os indivíduos no mesmo estágio fenológico, já que diversas espécies estudadas não têm ciclos regulares de florescimento. De qualquer forma, pudemos realizar comparações sobre todos os parâmetros da germinação de alface analisados através de valores únicos de IAs para cada espécie, através da dose resposta de quatro concentrações. O estabelecimento da dose resposta de três ou mais concentrações de material ou lixiviado fitotóxico mostra-se ideal para se obter dados confiáveis sobre a atividade alelopática (Inderjit \& Weston 2000). A necessidade da espécie alvo ser do mesmo ambiente do organismo doador é controversa. Plantas terrestres ou partes delas são algumas vezes utilizadas porque podem fornecer o melhor sistema modelo para uma detalhada investigação do modo de ação (Gross 2003).

O potencial osmótico das soluções pode ser um fator negativo adicional aos seus efeitos alelopáticos, reduzindo, atrasando ou impedindo a germinação (Astarita et al. 1996). Os valores de potencial osmótico dos extratos foliares testados variaram entre -0,017 e -0,98 MPa. Verificamos que as soluções com concentrações de PEG 6000 a partir de $-0,4 \mathrm{MPa}$ aumentaram significativamente a entropia informacional e a velocidade de germinação de alface, enquanto que a porcentagem de germinação foi reduzida a partir do potencial -0,6 $\mathrm{MPa}$. Os efeitos das soluções na concentração $10 \%$ de A. danaeifolium, B. monnieri, E. flavescens, E. montana, I. cairica e T. dominguensis, e da solução $5 \%$ de C. americanum, sobre a entropia informacional e a velocidade de germinação de alface se devem, em parte, ao efeito de seus potenciais osmóticos. A solução $10 \%$ de C. americanum apresentou o potencial osmótico mais discrepante $(-0,98 \mathrm{MPa})$ e seus efeitos sobre todos os parâmetros da germinação de alface se devem parcialmente aos efeitos da sua osmolaridade. A 
Tabela 3. Entropias informacionais de germinação de Lactuca sativa L. sob ação de extratos em diferentes concentrações das espécies doadoras e respectivos Índices Alelopáticos (IAs). Letras minúsculas iguais não diferem entre si dentro da mesma linha e letras maiúsculas iguais não diferem entre si, pela ANOVA e pós-teste de Tukey $(\mathrm{p}<0,05)$. x: não calculado. 0,0: controle. As espécies foram dispostas do maior para o menor IA.

\begin{tabular}{|c|c|c|c|c|c|c|c|c|c|c|c|c|c|}
\hline \multirow{3}{*}{$\begin{array}{l}\text { Espécie } \\
\text { Ipomoea cairica (L) Sweet }\end{array}$} & \multirow{3}{*}{$\begin{array}{c}\text { Família } \\
\text { Convolvulaceae }\end{array}$} & \multicolumn{10}{|c|}{ Concentração (\%) } & \multirow{2}{*}{\multicolumn{2}{|c|}{ IA }} \\
\hline & & \multicolumn{2}{|c|}{0} & \multicolumn{2}{|c|}{1,25} & \multicolumn{2}{|c|}{2,5} & \multicolumn{2}{|c|}{5} & \multicolumn{2}{|c|}{10} & & \\
\hline & & 0,5 & $\mathrm{~b}$ & 1,0 & $\mathrm{a}$ & 0,7 & a & 2,1 & $\mathrm{a}$ & $\mathrm{x}$ & & 10,8 & A \\
\hline Sagittaria montevidensis Cham. \& Schl. & Alismataceae & 0,5 & $\mathrm{~d}$ & 1,2 & $\mathrm{bc}$ & 0,9 & $\mathrm{~cd}$ & 2,7 & $\mathrm{a}$ & $\mathrm{x}$ & & 10,7 & A \\
\hline Crinum americanum $\mathrm{L}$. & Amaryllidaceae & 0,5 & $\mathrm{~b}$ & 1,2 & $\mathrm{~b}$ & 1,6 & $\mathrm{~b}$ & 1,6 & $\mathrm{a}$ & $\mathrm{x}$ & & 9,8 & $\mathrm{AB}$ \\
\hline Eleocharis flavescens (Poir.) Urb. & Cyperaceae & 0,5 & $\mathrm{~b}$ & 0,6 & $\mathrm{~b}$ & 1,1 & $\mathrm{~b}$ & 1,8 & $\mathrm{a}$ & $\mathrm{x}$ & & 9,3 & $\mathrm{AB}$ \\
\hline Ludwigia hyssopifolia (G. Don) Exel & Onagraceae & 0,5 & e & 0,7 & de & 1,5 & $\mathrm{~cd}$ & 2,0 & $\mathrm{bc}$ & 2,6 & a & 9,3 & $\mathrm{AB}$ \\
\hline Acroceras zizanioides (HBK) Dandy & Poaceae & 0,5 & $\mathrm{~d}$ & 0,7 & $\mathrm{~cd}$ & 1,3 & c & 1,7 & $\mathrm{~b}$ & 2,7 & a & 9,1 & $\mathrm{AB}$ \\
\hline Thelypteris interrupta (Willd.) Iwats. & Thelypteridaceae & 0,5 & c & 0,9 & c & 1,1 & $\mathrm{bc}$ & 1,9 & $\mathrm{~b}$ & 2,6 & a & 8,8 & $\mathrm{AB}$ \\
\hline Hymenachne amplexicaulis (Rudge) Ness. & Poaceae & 0,5 & c & 0,9 & $\mathrm{bc}$ & 1,4 & $\mathrm{ab}$ & 1,5 & $\mathrm{a}$ & 1,9 & a & 8,7 & $\mathrm{AB}$ \\
\hline Ludwigia octovalvis (Jacq.) Raven & Onagraceae & 0,5 & c & 0,3 & $\mathrm{c}$ & 1,3 & $\mathrm{~b}$ & 2,0 & $\mathrm{a}$ & 2,1 & a & 8,6 & $\mathrm{AB}$ \\
\hline Ludwigia erecta (L.) H. Hara & Onagraceae & 0,5 & $\mathrm{~b}$ & 0,6 & $\mathrm{~b}$ & 1,2 & $\mathrm{~b}$ & 2,2 & $\mathrm{a}$ & 2,6 & a & 8,5 & $\mathrm{AB}$ \\
\hline Bacopa monnieri (L.) Wettst. & Scrophulariaceae & 0,5 & c & 0,5 & c & 1,1 & $\mathrm{~b}$ & 1,3 & $\mathrm{~b}$ & 2,8 & a & 8,4 & $\mathrm{AB}$ \\
\hline Brachiaria mutica (Forsk) Stapf & Poaceae & 0,5 & c & 0,4 & $\mathrm{c}$ & 0,3 & c & 1,3 & $\mathrm{~b}$ & 2,1 & a & 8,2 & $\mathrm{AB}$ \\
\hline Acrostichum danaeifolium Langsd \& Fisch & Pteridaceae & 0,5 & c & 0,1 & c & 1,4 & $\mathrm{~b}$ & 2,2 & $\mathrm{a}$ & 1,9 & $\mathrm{ab}$ & 7,9 & $\mathrm{AB}$ \\
\hline Eleocharis interstincta (Vahl) Roem. \& Schult. & Cyperaceae & 0,5 & $\mathrm{~b}$ & 1,0 & $\mathrm{~b}$ & 1,3 & $\mathrm{~b}$ & 1,3 & $\mathrm{~b}$ & 2,4 & a & 7,7 & $\mathrm{AB}$ \\
\hline Typha dominguensis Pers. & Typhaceae & 0,5 & $\mathrm{~d}$ & 0,5 & $\mathrm{~d}$ & 1,0 & $\mathrm{~cd}$ & 1,4 & $\mathrm{ab}$ & 1,8 & a & 7,3 & $\mathrm{AB}$ \\
\hline Polygonum ferrugineum Wedd. & Polygonaceae & 0,7 & $\mathrm{~b}$ & 0,9 & $\mathrm{ab}$ & 0,8 & $\mathrm{~b}$ & 1,2 & a & 1,2 & a & 4,8 & $\mathrm{AB}$ \\
\hline Polygonum hydropiperoides Michx. & Polygonaceae & 0,7 & c & 0,6 & c & 0,5 & c & 1,2 & $\mathrm{~b}$ & 2,4 & a & 4,3 & $\mathrm{AB}$ \\
\hline Polygonum meisnerianum Cham. \& Schltdl. & Polygonaceae & 0,7 & c & 0,4 & c & 0,6 & c & 1,3 & $\mathrm{~b}$ & 1,9 & a & 4,0 & $\mathrm{AB}$ \\
\hline Scleria latifolia Sw. & Cyperaceae & 0,7 & $\mathrm{ab}$ & 0,6 & $\mathrm{~b}$ & 0,9 & $\mathrm{ab}$ & 1,2 & $\mathrm{a}$ & 1,0 & $\mathrm{ab}$ & 3,7 & $\mathrm{AB}$ \\
\hline Calyptrocarya longifolia (Rudge) Kunth & Cyperaceae & 0,7 & $\mathrm{~b}$ & 0,5 & $\mathrm{~b}$ & 0,7 & $\mathrm{ab}$ & 1,2 & a & 1,0 & $\mathrm{~b}$ & 3,3 & $\mathrm{AB}$ \\
\hline Echinochloa polystachya (Kunth) Hitchc & Poaceae & 0,5 & $\mathrm{bc}$ & 0,3 & $\mathrm{c}$ & 1,0 & $\mathrm{~b}$ & 0,8 & $\mathrm{bc}$ & 2,1 & a & 3,3 & $\mathrm{AB}$ \\
\hline Schoenoplectus californicus (C.A. Mey.) Soják & Cyperaceae & 0,5 & $\mathrm{~b}$ & 0,4 & $\mathrm{~b}$ & 0,5 & $\mathrm{~b}$ & 0,9 & $\mathrm{~b}$ & 2,6 & a & 3,2 & $\mathrm{AB}$ \\
\hline Eleocharis minima Kunth & Cyperaceae & 0,5 & $\mathrm{~b}$ & 0,8 & $\mathrm{ab}$ & 1,1 & a & 0,7 & $\mathrm{ab}$ & 0,7 & $\mathrm{ab}$ & 1,9 & $\mathrm{AB}$ \\
\hline Scleria mitis O. Berg & Cyperaceae & 0,7 & $\mathrm{~b}$ & 0,3 & $\mathrm{~b}$ & 0,6 & $\mathrm{~b}$ & 0,8 & $\mathrm{ab}$ & 1,3 & a & 0,9 & $\mathrm{AB}$ \\
\hline \multirow[t]{2}{*}{ Eleocharis montana (Kunth) Roem. \& Schult. } & Cyperaceae & 0,5 & $\mathrm{~b}$ & 0,3 & $\mathrm{~b}$ & 0,6 & $\mathrm{~b}$ & 0,6 & $\mathrm{~b}$ & 1,7 & a & 0,7 & $\mathrm{AB}$ \\
\hline & & & & & & & & & & & & 0,0 & B \\
\hline
\end{tabular}

germinação de sementes de alface não é afetada sob condições de escuridão/luminosidade dentro dos limites de $\mathrm{pH} 2,6$ a 10,6 (Reynolds 1975). O pH dos extratos foliares variou de 4,4 a 6,9 , descartando a possibilidade de efeitos significativos deste fator sobre a germinação.

Com base nos dados obtidos, concluímos que as espécies doadoras mostraram diferentes efeitos sobre o processo de germinação das sementes de alface. Das 25 espécies estudadas, 11 diminuíram a porcentagem de germinação, todas diminuíram a velocidade e aumentaram a entropia informacional de germinação das sementes da espécie alvo. O cálculo do IA para os parâmetros da germinação mostrou-se eficaz para ranquear as espécies doadoras de acordo com a curva dose-reposta. Ensaios em laboratório são importantes ferramentas para se obter um modelo do processo alelopático e nortear a seleção de espécies doadoras para experimentos com espécies do ambiente natural ou de sistemas agricultáveis. Porém, futuros estudos são necessários para comprovar essa interação em campo.

\section{Agradecimentos}

À Coordenação de Aperfeiçoamento de Pessoal de Nível Superior (CAPES) pela concessão da bolsa ao primeiro autor, e ao Conselho Nacional de Desenvolvimento Científico e Tecnológico (CNPq) pela concessão de bolsa ao segundo autor. 


\section{Referências bibliográficas}

Aliotta, G., Cafiero, G. \& Otero, A.M. 2006. Weed germination, seedling growth and their lesson for allelopathy in agriculture. Pp. 285-297. In: Reigosa, M.J.; Pedrol, N. and González, L. (Eds.). Allelopathy: A Physiological Process with Ecological Implications. Dordrecht, Springer.

An, M. 2005. Mathematical Modelling of Dose-Response Relationship (Hormesis) in Allelopathy and its Aplication. Nonlinearity in Biology, Toxicology, and Medicine Nonlinearity in Biology, Toxicology, and Medicine 3: 153-172.

Astarita, L.V., Ferreira, A.G. \& Bergonci, J.I. 1996. Mimosa bimucronata: Allelopathy and osmotic stress. Allelopathy Journal Allelopathy Journal 3(1): 43-50.

Blanco, J.A. 2007. The representation of allelopathy in ecosystem-level forest models. Ecological Modelling Ecological Modelling 209(24): 65-77.

Brasil. 1992. Regras para análise de sementes. Brasília, Ministério da Agricultura e Reforma Agrária.

Chou, C.H. 1989. Allelopathic research of subtropical vegetation in Taiwan. IV. Comparative phytotoxic nature of leachate from four subtropical grasses Journal of Chemical Ecology Journal of Chemical Ecology 15(7): 2149-2159.

Erhard, D. \& Gross, E.M. 2006. Allelopathic activity of Elodea canadensis and Elodea nuttalli against epiphytes and phytoplankton. Aquatic Botany Aquatic Botany 85: 203-211.

Esteves, F.A. 1988. Fundamentos de Limnologia. 2. ed. Rio de Janeiro, Editora Interciência.

Ferreira, A.G. \& Aquila, M.E.A. 2000. Alelopatia: Uma área emergente da ecofisiologia. Revista Brasileira de Fisiologia Vegetal Revista Brasileira de Fisiologia Vegetal 12: 175-204.

Gallardo, T.M., Martin, B.B. \& Martin, D.F. 1998. Inhibition of water fern Salvinia minima by cattail (Typha dominguensis) extracts and by 2-chlorophenol and salicylaldehyde. Journal of Chemical Ecology Journal of Chemical Ecology 24: 1483-1490.

Gross, E.M. 2003. Allelopathy of Aquatic Autotrophs. Critical Reviews in Plant Sciences Critical Reviews in Plant Sciences 22(3-4): 313-339.

Hierro, J.L. \& Callaway, R.M. 2003. Allelopathy and exotic plant invasion. Plant and Soil Plant and Soil 256: 29-39.

Hoagland, R.E. \& Williams, R.D. 2003. Bioassays: Useful tools for the study of allelopathy. Pp. 315-351. In: Macias, F.A.; Galindo, J.C.G.; Molinillo, J.M.G. and Cutler, H.G. (Eds.). Allelopathy: Chemistry and Mode of Action of Allelochemicals. Boca Raton, FL, CRC Press.

Inderjit \& Weston, L.A. 2000. Are laboratory biossays for allelopathy suitable for prediction of field responses? Journal of Chemical Ecology Journal of Chemical Ecology 26(9): 2111-2118.

Jeller, H. \& Perez, S.C.J.G.A. 2001. Efeito dos estresses hídrico e salino e da ação de giberelina em sementes de Senna spectabilis. Ciência Florestal Ciência Florestal 11(1): 93-104.
Labouriau, L.F.G. 1983. A germinação das sementes. Washington, Departamento de Assuntos Científicos e Tecnológicos da Secretaria Geral da Organização dos Estados Americanos.

Llamas, K.A. 2003. Tropical Flowering Plants: a guide to identification and cultivation 1. ed. Portland, Timber Press.

Lorenzi, H. 2000. Plantas daninhas do Brasil: terrestres, aquáticas, parasitas e tóxicas. 3. ed. Nova Odessa, Instituto Plantarum.

Ma, R.J., Wang, N.L., Zhu, H., Guo, S.J. \& Chen, D.S. 2009. Isolation and identification of allelochemicals from invasive plant Ipomoea cairica. Allelopathy Journal Allelopathy Journal 24(1): 77-84.

Macías, F.A., Oliva, R.M., Simonet, A.M. \& Galindo, J.C.G. 1998. What are allelochemicals? Pp. 154. In: Olofsdotter, M. (Ed.). Allelopathy in rice: Proceedings of the Workshop on Allelopathy in Rice. Manila (Philippines), International Rice Research Institute.

Maraschin-Silva, F. \& Aquila, M.E.A. 2006. Potencial alelopático de espécies nativas na germinação e crescimento inicial de Lactuca sativa $\mathrm{L}$. (Asteraceae). Acta Botanica Brasilica Acta Botanica Brasilica 20(1):61-69.

Otte, M.L. 2001. What is stress to a wetland plant? Environmental and Experimental Botany Environmental and Experimental Botany 46: $195-202$

Phillips, G.L., Eminson, D.F. \& Moss, B. 1978. A mechanism to account for macrophyte decline in progressively eutrophicated freshwater. Aquatic Botany Aquatic Botany 4: 103-126.

Putnam, A.R. \& Tang, C.S. 1986. Allelopathy: State of the science. Pp. 1-19. In: Putnam, A.R. and. Tang, C.S (Eds.). The science of allelopathy. New York, John Wiley \& Sons.

Reigosa, M.J., Sánchez-Moreiras, A. \& González, L. 1999. Ecophysiological Approach in Allelopathy Critical Reviews in Plant Sciences Critical Reviews in Plant Sciences 14(5): 577-608.

Reynolds, T. 1975. pH restraints on lettuce fruit germination. Annals of Botany Annals of Botany 39: 797-805.

Ribeiro, J.P.N., Matsumoto, R.S., Takao, L.K., Voltarelli, V.M. \& Lima, M.I.S. 2009. Efeitos alelopáticos de extratos aquosos de Crinum americanum $\mathrm{L}$. Revista Brasileira de Botânica Revista Brasileira de Botânica 32(1): 183-188.

Rice, E.L. 1984. Allelopathy. 2. ed. Orlando, Academic Press.

Souza Filho, A.P.S., Alves, S.M. \& Dutra, S. 2002. Development Stages and Water Stress on the Allelopathic Potential of Marandu Grass. Planta Daninha Planta Daninha 20(1): 25-31.

Sutton, D.L. \& Portier, K.M. 1991. Influence of Spikerush Plants on Growth and Nutrient Content of Hydrilla. Journal of Aquatic Plant Manage Journal of Aquatic Plant Manage 29: 6-11.

Villela, F.A., Doni Filho, L. \& Sequeira, E.L. 1991. Tabela de potencial osmótico em função da concentração de polietileno glicol 6000 e da temperatura. Pesquisa Agropecuária Brasileira Pesquisa Agropecuária Brasileira 26(11/12): 1957-1968.

Wolanski, E. 2007. Estuarine Ecohydrology. 1. Amsterdam, Elsevier Science Ltd.

Wooten, J.W. \& Elakovich, S.D. 1991. Comparison of potencial allelopathy of seven freshwater species of spikerushes (Eleocharis). Journal of Aquatic Plant Manage Journal of Aquatic Plant Manage 29: 12-15. 